SHORT REPORT

\title{
Fatal toxic leukoencephalopathy: clinical, radiological, and necropsy findings in two patients
}

\author{
A Ryan, F M Molloy, M A Farrell, M Hutchinson
}

J Neurol Neurosurg Psychiatry 2005;76:1014-1016. doi: 10.1136/jnnp.2004.047134

Background: Toxic leukoencephalopathy has been described with inhalation and intravenous consumption of heroin and cocaine. The clinical picture varies widely but the imaging and histological features are characteristic. Magnetic resonance imaging (MRI) typically reveals diffuse bihemispheric white matter lesions. Histologically there is extensive spongiform degeneration of the cerebral white matter.

Objective: To report two cases of fatal toxin associated leukoencephalopathy, along with detailed imaging and neuropathological studies.

Results: MRI revealed diffuse white matter changes. Histologically there was widespread confluent vacuolar degeneration of the deep white matter. In both cases, there was sparing of the brain stem and cerebellar white matter. There was evidence of severe and extensive axonal injury. Conclusions: This pattern of radiological involvement and histological findings has not previously been reported and may reflect the presence of a yet unidentified impurity.

$\mathrm{T}$ oxic leukoencephalopathy has been described in association with a wide variety of agents including cranial irradiation, environmental toxins, therapeutic drugs, and drugs of abuse. ${ }^{1}$ Inhalation of heroin vapour in particular leads to characteristic imaging and brain biopsy appearances. $^{2-4}$ Similar findings have been reported in cases of cocaine abuse. ${ }^{5}$ The clinical course is varied and ranges from an unexpectedly favourable outcome in some cases to rapid progression to death in others. ${ }^{2}$

Here we report two patients with a rapidly fatal toxic leukoencephalopathy. The patterns of radiological involvement as well as the neuropathological findings were strikingly similar to one another but could readily be distinguished from findings in previous reports of heroin and cocaine induced leukoencephalopathy. We speculate on the aetiology of leukoencephalopathy in these cases.

\section{CASE REPORTS}

\section{Case 1}

A 55 year old man presented with a two week history of behavioural change, worsening confusion, aggressive outbursts, and deficits in his short term memory. His relatives provided a history of cocaine abuse. On admission, he was disoriented in time, had a poor attention span, and was uncooperative and aggressive. He could follow a one step command. Deep tendon reflexes were brisk, with bilateral extensor plantar responses. Extraocular movements were full with no nystagmus. Over the next few days, his condition rapidly deteriorated. He became progressively drowsy and stuporose, with development of myoclonic jerks in all four limbs. His condition further declined and he became unresponsive, with the maintenance of a decorticate posture at rest. He died two weeks after admission.

\section{Case 2}

A 36 year old man was found unresponsive, with a Glasgow coma scale score of 3/15. There was no past medical history of note but he was known to have a history of substance abuse. At the time of admission, his eyes were in the mid-position of gaze and with pinpoint pupils. Deep tendon reflexes were generally brisk and plantar responses were extensor bilaterally. He remained unresponsive but was noted to have spontaneous eye opening on day 9 following admission. He then developed persistent pyrexia with methicillin resistant $S$ aureus septicaemia and died one month after admission.

\section{INVESTIGATIONS}

\section{Radiological findings}

Magnetic resonance imaging (MRI) of the brain was done in each case and showed diffuse bilateral cerebral white matter changes involving the frontal, parietal, occipital, and temporal lobes. The abnormalities were best appreciated on the fluid attenuated inversion recovery (FLAIR) sequence (fig 1 , panels $\mathrm{A}$ and $\mathrm{B}$ ). Changes were more extensive in the case of patient 1 . Of note, there was relative sparing of the subcortical U fibres, and the brain stem and cerebellar white matter were not affected. No contrast enhancement was seen following administration of a paramagnetic agent.

\section{Laboratory findings}

Results of haematological and biochemical tests for both patients-including complete blood count, serum electrolytes, liver function tests, ammonia, lactate, and pyruvatewere unremarkable. Toxicology screening of serum and urine was negative in both cases but was done some time after the last reported substance misuse. Cerebrospinal fluid (CSF) examination including cytological analysis was also normal. Additional investigations including serological tests for an infective agent such as human immunodeficiency virus (HIV), herpes simplex virus, JC virus, treponema pallidum, and borrelia were also negative, as were CSF viral and bacterial cultures. In the case of patient 1 , a vasculitic screen and magnetic resonance angiography were normal. There was no family history of a metabolic disorder. Urinary organic acids, very long chain fatty acids, and a porphyria screen were all within normal limits.

\section{Neuropathological findings}

In both cases there was profound vacuolar degeneration of the deep white matter involving the centrum semi-ovale of the two hemispheres. There was relative sparing of the subcortical $U$ fibres but the changes became progressively more severe from superficial to deep (fig 2, panels A and B). Cerebellar and brain stem white matter were not involved (fig 2D). In addition, there was evidence of severe axonal injury in the form of axonal spheroids and accumulation of 

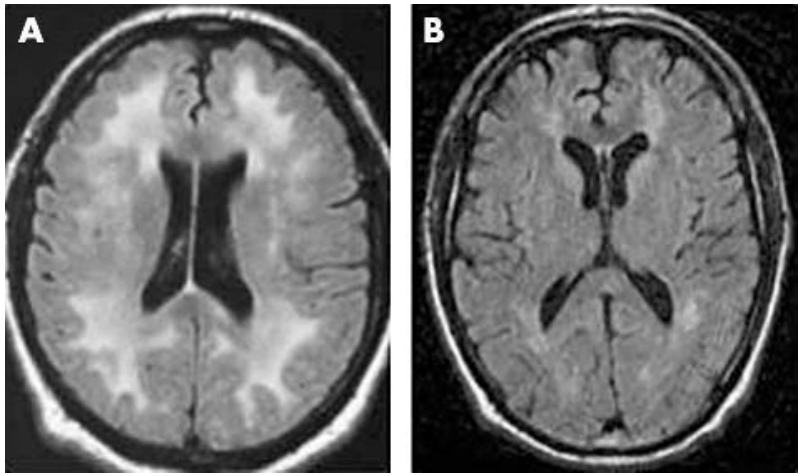

Figure 1 Axial fluid attenuated inversion recovery (FLAIR) magnetic resonance imaging sequences of the brain from patient $1(A)$ and patient 2 (B). Both scans show evidence of diffuse symmetrical white matter changes involving the periventricular region and extending superiorly to involve the corona radiata.

amyloid precursor protein positive axonal debris (fig 3). Electron microscopy revealed splitting and unravelling of the myelin sheath, extensive axonal loss, and axonal swelling. Blood vessels were normal and there was no evidence of any inflammatory or infective process.

\section{DISCUSSION}

The first report of leukoencephalopathy in association with heroin inhalation was by Wolters et al in 1982. ${ }^{2}$ Subsequent cases have been described across Europe and the USA, including an association with the intravenous use of cocaine as well as of heroin. ${ }^{5}$ The clinical presentation varies widely. There may be an altered level of consciousness, spasticity, a cerebellar syndrome, or the development of a bradykinetic rigid state. In some cases, there is rapid progression to death, but complete recovery may occur. ${ }^{5}$ We report two cases of leukoencephalopathy characterised by the development of a stuporose state which, in the case of patient 1 , was followed by a period of motor excitability before rapid progression to coma and death.

The neuroimaging findings of diffuse symmetrical white matter involvement, with sparing of the subcortical U fibres, are consistent with previous reports. ${ }^{67}$ A notable feature of the cases reported here is the complete absence of cerebellar and brain stem involvement. The involvement of the cerebellar and posterior cerebral white matter and the posterior limb of the internal capsule are typical features of heroin vapour inhalation ${ }^{6}$ but may not be specific for other toxic leukoencephalopathies. Alternatively, the particular adulterant in these two cases may spare the brain stem and cerebellum and preferentially target the cerebral white matter.

The histological findings in these two cases were almost identical. There was widespread confluent vacuolar degeneration of the deep white matter, with profound axonal loss and evidence of axonal injury in adjacent normal appearing white matter. It is therefore tempting to suggest that the axonal injury preceded the myelin vacuolar change. For the most part, however, the axonal injury and vacuolar change
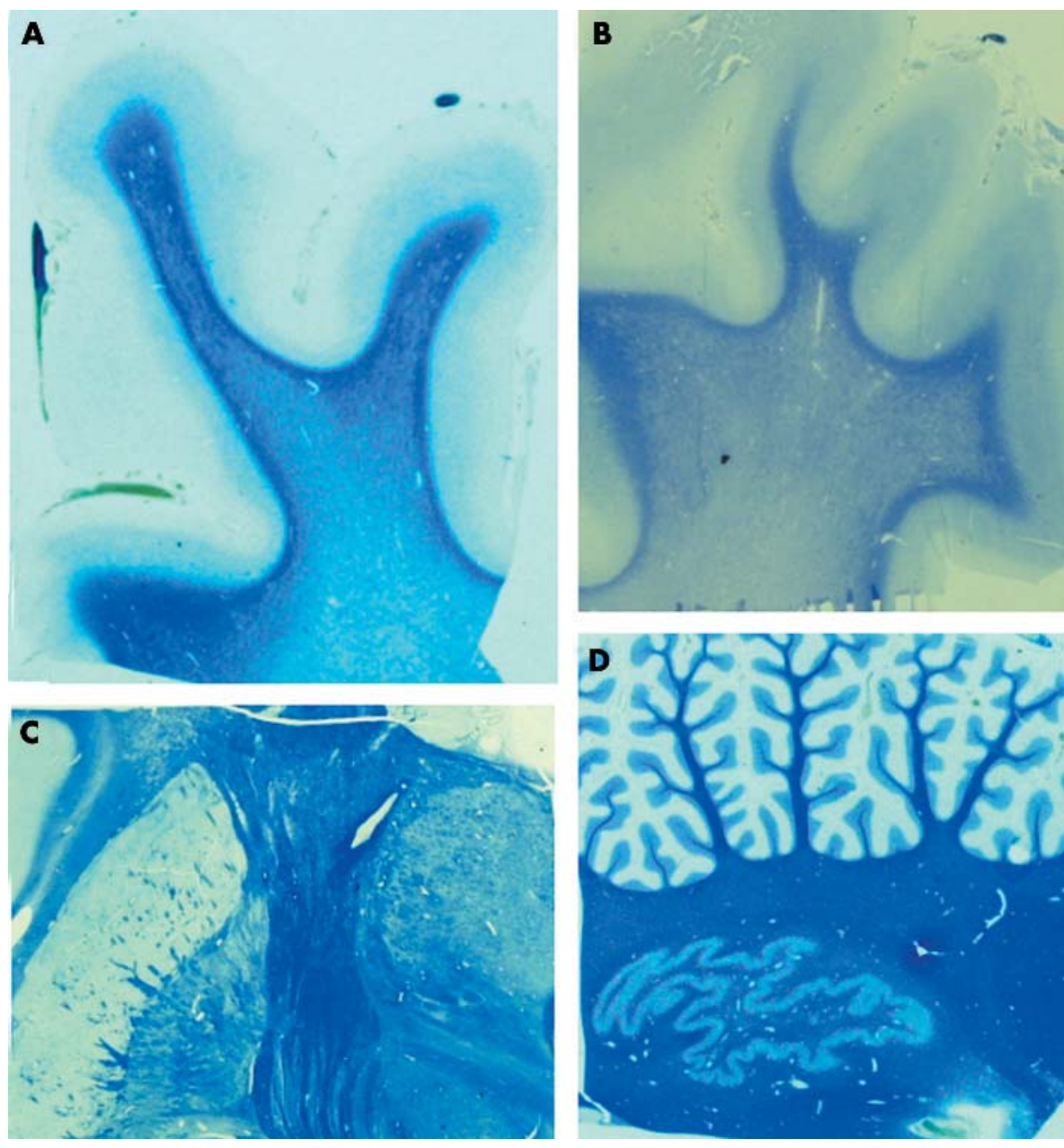

Figure 2 Myelin pallor in frontal (A) and parietal (B) white matter, contrasted with normal myelin staining intensity in internal capsule (C) and cerebellum (D). Note preservation of subcortical $U$ fibres in the frontal and parietal white matter. (All stained with Luxol-Fast Blve.)

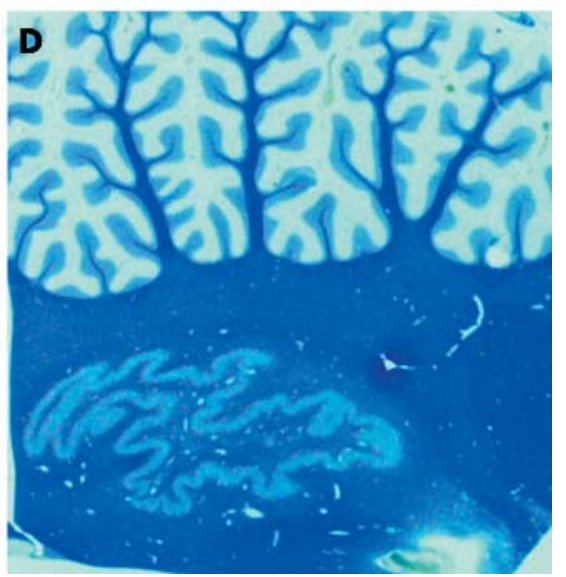



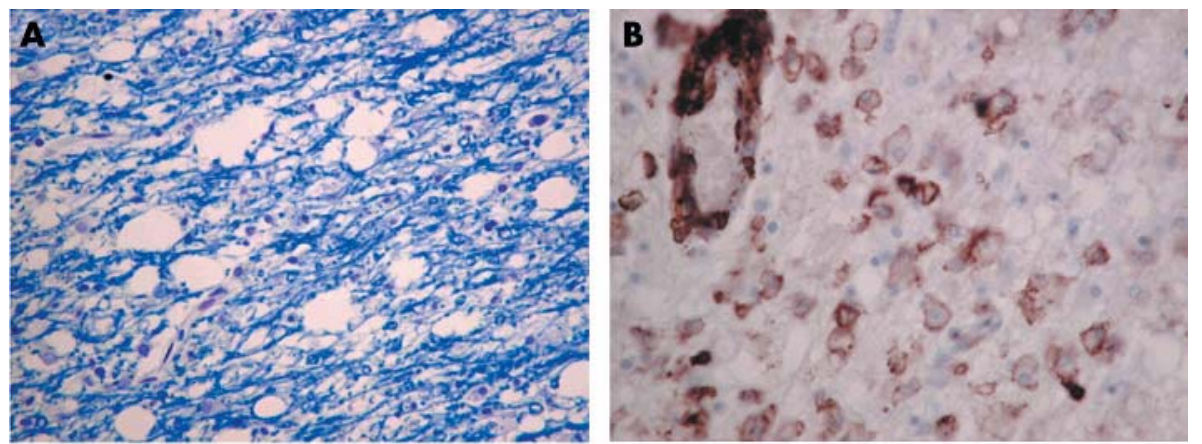

Figure 3 Vacuolar change deep frontal white matter (A) (Luxol-Fast Blue) associated with variable macrophage infiltrate (B) (common leukocyte immunostain); axonal loss (C) (neurofilament) and degenerating axons with spheroid formation (D) (amyloid precursor protein).
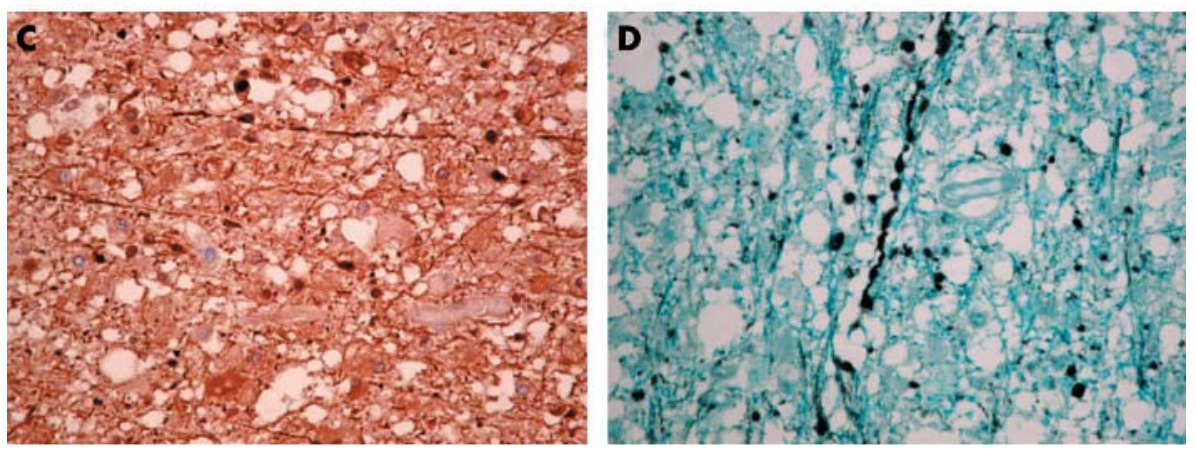

appeared to parallel each other. Such prominent axonal involvement is unusual in heroin induced leukoencephalopathy where axons are typically preserved. ${ }^{7}$

The aetiology of this condition is poorly understood. A defect in mitochondrial function has been suggested in two cases of leukoencephalopathy associated with heroin vapour inhalation where increased white matter lactate and a favourable response to antioxidants have been reported. ${ }^{7}$ In addition to a direct toxic effect on myelin and mitochondrial dysfunction, hypoxic injury may also contribute to the axonal damage and spongiform white matter change. ${ }^{8}$ There may be a latent period between toxin exposure and the development of symptoms and signs-a phenomenon referred to as "coasting", which has been described for several other neurotoxins. ${ }^{9}$ This may account for the negative toxicology result in each of these cases. It is thought that the toxic substance may be deposited in lipid-rich myelin where it resides and is slowly released, resulting in ongoing tissue destruction and symptom progression. Alternatively, the toxin may induce a metabolic change that persists following withdrawal of the offending substance. By the time the patient becomes symptomatic, the toxin is no longer detectable in the blood stream.

Toxic leukoencephalopathy caused by substance abuse has a wide clinical spectrum. The fatal outcome in each of these cases illustrates the potential seriousness of the condition. The pathology and imaging findings were strikingly similar to one another and resemble those of heroin inhalation toxicity but have several important distinguishing features, namely sparing of the cerebellum and brain stem, frontal rather than occipital predominance, and the more prominent axonal involvement. This may reflect the presence of a yet unidentified impurity. Despite the widespread use of cocaine, heroin, and other substances, with varying degrees of purity, these reported cases remain few and far between. Lack of awareness of this condition may lead to delayed or misdiagnosis. This is of potential clinical importance, particularly if treatment with antioxidants could lead to clinical improvement. Given current drug practices, it is likely that further cases will be identified.

\section{Authors' affiliations \\ A Ryan, F M Molloy, M Hutchinson, Department of Neurology, St Vincent's University Hospital, Dublin, Republic of Ireland M A Farrell, Department of Neuropathology, National Institute for Neurology and Neurosurgery, Beaumont Hospital, Dublin \\ Competing interests: none declared}

Correspondence to: Professor M Hutchinson, Department of Neurology, St Vincent's University Hospital, Elm Park, Dublin 4, Republic of Ireland; michael.hutchinson@svcpc.ie

Received 2 August 2004

In revised form 17 October 2004

Accepted 29 October 2004

\section{REFERENCES}

1 Filley CM, Kleinschmidt-DeMasters BK. Toxic leukoencephalopathy N Engl J Med 2001;345:425-32.

2 Wolters EC, van Wijngaarden GK, Stam FC, et al. Leucoencephalopathy after inhaling "heroin" pyrolysate. Lancet 1982;2:1233-7.

3 Celius EG, Andersson S. Leukoencephalopathy after inhalation of heroin: a case report. J Neurol Neurosurg Psychiatry 1996;60:694-5.

4 Weber W, Henkes H, Moller P, et al. Toxic spongiform leukoencephalopathy after inhaling heroin vapour. Eur Radiol 1998;8:749-55.

5 Maschke M, Fehlings T, Kastrup $O$, et al. Toxic leukoencephalopathy after intravenous consumption of heroin and cocaine with unexpected clinical recovery. J Neurol 1999;246:850-1.

6 Keogh CF, Andrews GT, Spacey SD, et al. Neuroimaging features of heroin inhalation toxicity: "chasing the dragon". Am J Roentgenol 2003; 180:847-50

7 Kriegstein AR, Shungu DC, Millar WS, et al. Leukoencephalopathy and raised brain lactate from heroin vapor inhalation ("chasing the dragon"). Neurology 1999;53:1765-73.

8 Vella S, Kreis R, Lovblad KO, et al. Acute leukoencephalopathy after inhalation of a single dose of heroin. Neuropediatrics 2003;34:100-4.

9 Berger AR, Schaumburg HH, Schroeder C, et al. Dose response, coasting, and differential fiber vulnerability in human toxic neuropathy: a prospective study of pyridoxine neurotoxicity. Neurology 1992;42:1367-70. 